\section{Mobile phones create new opportunities for microbiology research and clinical applications}
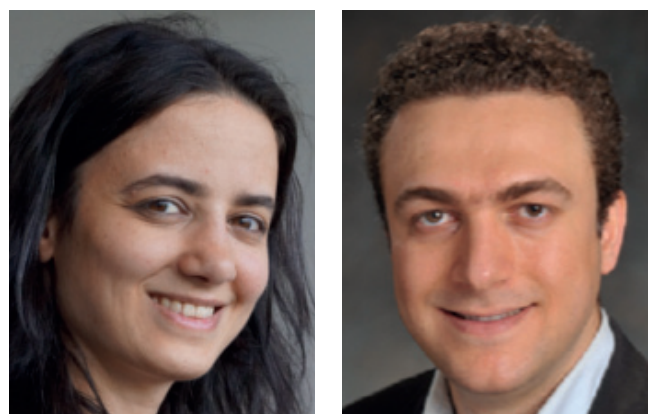

"...mobile phones have the potential to transform traditional uses of imaging, sensing and diagnostic systems, especially for pointof-care applications and field settings.”

Hatice Ceylan Koydemir ${ }^{1}$ \& Aydogan Ozcan ${ }^{*, 1,2,3}$

First draft submitted: 12 March 2017; Accepted for publication: 23 March 2017; Published online: 25 May 2017

Mobile phone-enabled measurement tools for research \& clinical use

Smartphones have been in our lives since the beginning of 2000s. By the end of 2015, 2 billion people across the world had a smartphone and the penetration rate of smartphones is expected to further increase to cover approximately $80 \%$ of the world population by 2020 [1]. Smartphones are equipped with a variety of advanced sensors such as digital cameras, microphones and accelerometers, among others [2]. Together with their cost-effectiveness, wide-spread use, computational power and connectivity, mobile phones have the potential to transform traditional uses of imaging, sensing and diagnostic systems, especially for point-of-care applications and field settings.

In fact, mobile phone-based imaging and sensing platforms have already been used in a variety of applications, including clinical chemistry, biomedical and environmental monitoring [3-5], food analysis [6-8], detection of different types of chemical and biological analytes such as cells, parasites [9], bacteria [10], eggs [11], proteins [12], various biomarkers [6,7], nanoparticles [13] and even nucleic acids [14-16]. In many of these platforms, processing of the acquired data and the resulting computational analysis are done either on the smartphone or over a local or remote server using a customdeveloped smartphone application. In some cases, subcomponents of the mobile phone peripherals, for example, the complementary metal-oxide-semiconductor (CMOS) imager chip of the camera module, can also be used for advanced microscopy and cytometry applications [17-19], in which case a laptop computer or a tablet can be used for image reconstruction and related analysis.

\section{KEYWORDS}

- biosensors • flow cytometry

- fluorescence microscopy • imaging

- mobile-phone microscopy • sensing

- smartphone-based instrumentation 


\section{"Different microscopic imaging modalities have been incorporated with mobile phones to provide field portable sensing platforms that can potentially be used for early detection and prevention of a variety of health problems."}

\section{Mobile phone-based microscopy, sensing \& diagnostics}

Different microscopic imaging modalities have been incorporated with mobile phones to provide field-portable sensing platforms that can potentially be used for early detection and prevention of a variety of health problems. Among these, smartphone-based fluorescence microscopy is one of the most commonly used imaging methods. The design of these compact, mobile phone-based microscopy tools generally contains a 3D printed opto-mechanical attachment unit, an external lens for magnification, an excitation filter and/or an emission filter, lightemitting diodes (LEDs) or laser diodes forming the excitation source, a translation stage to adjust the field-of-view and focus, and a sample tray. As an example, using a mobile-phone fluorescence microscope Zhu et al. [10] reported a quantum dot-based Escherichia coli detection platform integrated with glass-based capillary channels that were coated with antibodies specific to $E$. coli. This platform provided a limit of detection of 5-10 CFU per $\mathrm{ml}$ for fat-free milk samples contaminated with $E$. coli. In another study, a mobile phone-based fluorescence microscope was used as an imaging flow cytometer to analyze blood samples and biological contamination levels of drinking water samples [20]. In this design, the excitation of the fluorescent tags was done using LEDs and video capturing feature of the mobile phone was used to image fluorescently-labeled particles flowing in polydimethylsiloxane-based microfluidic channels. Another mobile phone-based imaging platform enabled rapid and sensitive detection and automated counting of waterborne pathogens (e.g., Giardia lamblia cysts) in large volume water samples (e.g., $10 \mathrm{ml}$ ) using image-processing algorithms and machine learning [4]. This design is composed of a smartphone-based fluorescence microscope, a disposable sample-processing cassette and a custom-developed smartphone application. Total time to result, including sample collection, labeling, filtration, optical detection and automated counting, is approximately $1 \mathrm{~h}$, which is significantly shorter compared with conventional methods [21] which can take more than $24 \mathrm{~h}$. This handheld and cost-effective platform weighs only approximately $205 \mathrm{~g}$ and provides a limit of detection of approximately 12 cysts per $10 \mathrm{ml}$ with a $G$. lamblia cyst classification accuracy of approximately $95 \%$ for nonpotable, pond, tap and reagent grade water samples.
Smartphone-based fluorescence microscopes have also been used for imaging nano-sized biological specimen $[13,15,16]$. In the design of these microscopes, a laser diode-based illumination with a highly-oblique incidence angle (e.g., $\sim 75^{\circ}$ ) is used for excitation of target fluorescent tags and particles, which provides a strong rejection of the excitation beam due to the low numerical aperture of the mobile phone camera system. Based on this design, isolated $100 \mathrm{~nm}$ fluorescent particles and human cytomegaloviruses were imaged using a cost-effective smartphone attachment weighing only approximately $186 \mathrm{~g}$ [13]. A similar design makes it possible to image and measure the lengths of individual double-stranded DNA molecules with a sizing accuracy of $<1000$ base pairs (stretched on a surface and labeled with YOYO-1 iodide) using a smartphone [15]. This mobile phone-enabled DNA imaging and sizing platform can serve as a useful point-of-care tool to detect antimicrobial drug resistance related genes that cause difficulty to treat infections in humans.

Smartphones have also been used as colorimetric readers $[3,7,22]$, for example, to test antimicrobial drug susceptibility [23] and analyze ELISA-based tests, such as mumps immunoglobulin, measles IgG and herpes simplex virus IgG tests, with a very similar level of performance compared with US FDA-approved clinical well-plate readers [3]. This mobile phone-based 96-well reader design $[3,7]$ is composed of a 3D printed opto-mechanical attachment unit that houses 96 multi-mode plastic fiber-optic cables that are each coupled to individual wells of a disposable well plate, enabling the measurement of the transmitted light from the wells, all in parallel and without the need for any mechanical scanning. In this handheld well-plate reader, the wells are illuminated using an array of LEDs and the uniformity of the illumination is ensured using an optical diffuser. Quantified analysis of the optical signal that is carried with the fiber-optic array is rapidly performed over a server (e.g., a simple laptop) using a machine learning-based image analysis algorithm without the need for an expert reader. This mobile phone and cost-effective well-plate reader enables highthroughput analysis of biological samples for many patients at once.

Another important imaging modality that is integrated with smartphones is bright-field microscopy. In this mode of imaging, a sample of interest is illuminated uniformly using white light (e.g., a broadband LED) and imaged through the 
camera of a smartphone together with an external lens that is used for magnification. This lowcost design can be used to detect pathogens of a variety of diseases, even at low-resource settings. For example, D’Ambrosio et al. [9] developed a mobile phone-based video microscope to detect and automatically quantify Loa loa microfilariae in whole-blood samples without any labeling steps. In this design, an LED array illuminates the whole-blood sample in capillaries and the microfilaria in blood are then detected by processing the acquired sequential frames of each field of view of the sample, with the results provided in less than $2 \mathrm{~min}$. Field testing of this prototype was conducted in Cameroon, Central Africa using 33 Loa-infected patients, demonstrating 94\% specificity and $100 \%$ sensitivity in comparison to manual thick smear counts. Another recent study [11] evaluated the performance of a smartphone-based bright-field microscope for labelfree detection of Schistosoma spp. eggs in urine samples in rural areas of Accra, Ghana, Africa. This cost-effective and field-portable smartphone-based microscope has a spatial resolution of approximately $0.87 \mu \mathrm{m}$ and uses a translation stage to scan a microscope slide for the presence of Schistosoma haematobium eggs. The field experiments conducted with this mobile phone microscope revealed a sensitivity of $72.1 \%$, a specificity of $100 \%$, a positive-predictive value of $100 \%$ and a negative-predictive value of $57.1 \%$ [11] .

\section{Commercialization of mobile phone- based measurement tools}

In addition to these proof-of-concept experiments as well as clinical validation and related testing, there are several smartphone-based biomedical measurement tools that are commercially available. As an example, Mudanyali et al. [5] devised a mobile phone-based transmission and reflection imaging system to provide a field portable and cost-effective rapid diagnostic test reader. Using this mobile reader, an image of a rapid diagnostic test is captured and digitally processed on the phone for automated reading and quantification of the test results through a custom-developed smartphone application. This mobile phonebased imaging platform has been commercialized by Holomic/Cellmic, LLC (CA, USA), and is currently in use in several countries for quantification of various colorimetric and fluorometric tests. Another commercially available mobile phone-based reflection microscope is released by MetaOptima Technology, Inc. (Vancouver,
BC, Canada) under the name of MoleScope [24]. This mobile phone-based imaging device enables capturing of high quality images of the skin (e.g., moles) and share them with medical professionals through an application.

\section{Future perspective}

The above outlined mobile phone-based imaging, sensing and diagnostic tools, together with many others, might be transformative to bring advanced biomedical measurements and testing to resource poor settings, point-of-care offices and even to our homes, and will get more and more powerful with the emergence of internet of things, 5G networks and edge computing. All of these will provide extreme connectivity and access to advanced computational resources, including machine learning-based data analytics that will be used to harness the resulting microscopic imaging, sensing and diagnostics data generated by such mobile measurement tools. These capabilities will provide unique opportunities and a much needed toolset especially for microbiology research, preventive and precision medicine as well as microbiome initiatives.

\section{Financial \& competing interests disclosure}

The Ozcan Research Group at UCLA gratefully acknowledges the support of the Presidential Early Career Award for Scientists and Engineers (PECASE), the Army Research Office (ARO; W911NF-13-1-0419 and W911NF-13-1-0197), the ARO Life Sciences Division, the National Science Foundation (NSF) CBET Division Biophotonics Program, the NSF Emerging Frontiers in Research and Innovation (EFRI) Award, the NSF EAGER Award, NSF INSPIRE Award, NSF Partnerships for Innovation: Building Innovation Capacity (PFI:BIC) Program, Office of Naval Research (ONR), the National Institutes of Health (NIH), the Howard Hughes Medical Institute (HHMI), Vodafone Americas Foundation, the Mary Kay Foundation, Steven \& Alexandra Cohen Foundation, and KAUST. This work is based upon research performed in a laboratory renovated by the National Science Foundation under Grant No. 0963183, which is an award funded under the American Recovery and Reinvestment Act of 2009 (ARRA). A Ozcan is the founder of Holomic/Cellmic LLC that commercializes mobile microscopy and sensing technologies. The authors have no other relevant affliations or financial involvement with any organization or entity with a financial interest in or financial conflict with the subject matter or materials discussed in the manuscript apart from those disclosed.

No writing assistance was utilized in the production of this manuscript.
“This low-cost design can be used to detect

pathogens of a variety of diseases, even at low-resource settings." 


\section{References}

1 Economist. Planet of the phones (2015). www.economist.com/news/leaders/21645180

2 Ozcan A. Mobile phones democratize and cultivate next-generation imaging, diagnostics and measurement tools. Lab Chip 14(17), 3187-3194 (2014).

3 Berg B, Cortazar B, Tseng D et al. Cellphonebased hand-held microplate reader for point-of-care testing of enzyme-linked immunosorbent assays. ACS Nano 9(8), 7857-7866 (2015).

4 Koydemir HC, Gorocs Z, Tseng D et al. Rapid imaging, detection and quantification of Giardia lamblia cysts using mobile-phone based fluorescent microscopy and machine learning. Lab Chip 15(5), 1284-1293 (2015).

5 Mudanyali O, Dimitrov S, Sikora U, Padmanabhan S, Navruz I, Ozcan A. Integrated rapid-diagnostic-test reader platform on a cellphone. Lab Chip 12(15), 2678-2686 (2012).

6 Ludwig SKJ, Tokarski C, Lang SN et al. Calling biomarkers in milk using a protein microarray on your smartphone. PLoS ONE 10(8), e0134360 (2015).

7 Ludwig SKJ, Zhu H, Phillips S et al. Cellphone-based detection platform for rbST biomarker analysis in milk extracts using a microsphere fluorescence immunoassay. Anal. Bioanal. Chem. 406(27), 6857-6866 (2014).

8 Coskun AF, Wong J, Khodadadi D, Nagi R, Tey A, Ozcan A. A personalized food allergen testing platform on a cellphone. $L a b$ Chip 13(4), 636-640 (2013).

9 D’Ambrosio MV, Bakalar M, Bennuru S et al. Point-of-care quantification of blood-borne filarial parasites with a mobile phone microscope. Sci. Transl. Med. 7(286), 286re4 (2015).

10 Zhu H, Sikora U, Ozcan A. Quantum dot enabled detection of Escherichia coli using a cell-phone. Analyst 137(11), 2541 (2012).

11 Bogoch II, Koydemir HC, Tseng D et al. Evaluation of a mobile phone-based microscope for screening of Schistosoma haematobium infection in rural Ghana. Am. J. Trop. Med. Hyg. 10 (6), e0004768 (2017).

12 Coskun AF, Nagi R, Sadeghi K, Phillips S, Ozcan A. Albumin testing in urine using a smart-phone. Lab Chip 13(21), 4231-4238 (2013).

13 Wei Q, Qi H, Luo W et al. Fluorescent imaging of single nanoparticles and viruses on a smart phone. ACS Nano 7(10), 9147-9155 (2013).

14 Feng S, Tseng D, DiCarlo D, Garner O, Ozcan A. High-throughput and automated diagnosis of antimicrobial resistance using a cost-effective cellphone-based micro-plate reader. Sci. Rep. 6, 39203 (2016).

15 Wei Q, Luo W, Chiang S et al. Imaging and sizing of single DNA molecules on a mobile phone. ACS Nano 8(12), 12725-12733 (2014).

16 Kühnemund M, Wei Q, Darai E et al. Targeted DNA sequencing and in situ mutation analysis using mobile phone microscopy. Nat. Commun. 8, 13913 (2017).
17 Wei Q, McLeod E, Qi H, Wan Z, Sun R, Ozcan A. On-chip cytometry using plasmonic nanoparticle enhanced lensfree holography. Sci. Rep. 3, 1699 (2013).

18 Tseng D, Mudanyali O, Oztoprak C et al. Lensfree microscopy on a cellphone. Lab Chip 10(14), 1787-1792 (2010).

19 Mudanyali O, Tseng D, Oh C et al. Compact, light-weight and cost-effective microscope based on lensless incoherent holography for telemedicine applications. Lab Chip 10(11), 1417-1428 (2010).

20 Zhu H, Mavandadi S, Coskun AF, Yaglidere O, Ozcan A. Optofluidic fluorescent imaging cytometry on a cell phone. Anal. Chem. 83(17), 6641-6647 (2011).

21 EPA. Method 1623.1: Cryptosporidium and Giardia in water by filtration/IMS/FA. http://water.epa.gov/scitech/drinkingwater

22 Vashist SK, van Oordt T, Schneider EM, Zengerle R, von Stetten F, Luong JHT. A smartphone-based colorimetric reader for bioanalytical applications using the screen-based bottom illumination provided by gadgets. Biosens. Bioelectron. 67, 248-255 (2015).

23 Feng S, Tseng D, Carlo DD, Garner OB, Ozcan A. High-throughput and automated diagnosis of antimicrobial resistance using a cost-effective cellphone-based micro-plate reader. Sci. Rep. 6, 39203 (2016).

24 MetaOptima. MoleScope. https://molescope.com/product 